\title{
Videometrics-based Detection of Vibration Linearity in MEMS Gyroscope
}

\author{
Yong Zhou*, Haigang Sun, Shiqiao Gao \\ School of Mechanical Engineering, Beijing Institute of Technology \\ Beijing 100081, China \\ Shaohua Niu \\ Department of Civil and Environmental Engineering Sciences, Rulr-University Bochum \\ Bochum 44780, Germany \\ Received: 24-02-2011 \\ Accepted: 18-04-2011
}

\begin{abstract}
MEMS gyroscope performs as a sort of sensor to detect angular velocity, with diverse applications in engineering including vehicle and intelligent traffic etc. A balanced vibration of driving module excited by electrostatic driving signal is the base MEMS gyroscope's performance. In order to analyze the linear property of vibration in MEMS Gyroscope, a method of computer vision measuring is applied with the help of high-speed vidicon to obtain video of linear vibration of driving module in gyroscope, under the driving voltage signal of inherent frequency and amplitude linearly increasing. By means of image processing, target identifying, and motion parameter extracting from the obtained video, vibration curve with time variation is acquired. And then, linearity of this vibration system can be analyzed by focusing on the amplitude value of vibration responding to the amplitude variation of driving voltage signal.
\end{abstract}

Keywords: MEMS gyroscope, videometrics, vibration system, linearity.

\section{Introduction}

MEMS gyroscope performing as a sort of sensor to detect angular velocity possesses many excellent characters, such as small size, little weight, low power loss, high capacity for resisting over loading, and finer environment adaptability. It has diverse applications in engineering including vehicle, intelligent traffic, and nanotechnology etc. Linear vibration MEMS gyroscope, which performs in different principium from traditional gyroscope, works in this way that the driving module is kept a balanced linear vibration in $\mathrm{x}$ axis direction, an angular velocity on $\mathrm{z}$ axis could be detected by Coriolis force measuring in y axis direction. So stability and linearity of the vibration of driving module is the predominant factor for angular velocity detection and gyroscope's performance. In order to evaluate the linearity of the vibration system of driving module in working state, this paper initiates a particular method of computer vision measuring system. Firstly, motion video of the vibration in gyroscope is obtained by high-speed vidicon under the condition that the driving module is impelled by driving voltage signal of inherent frequen-

\footnotetext{
* Corresponding author: bitzhouy@bit.edu.cn
} 
cy and amplitude linearly increasing. This motion video represents the displacement of driving detecting comb and their joint micro-structure in gyroscope corresponding to the impelling voltage signal. Secondly, the vibration curve with time variation can be acquired by means of image processing, target identifying and motion parameter extracting from the obtained video in computer image processing system. And then, relationship between the amplitude of this vibration and the amplitude of driving voltage signal can be regarded as response output and its input for a testing system ${ }^{1}$, and the linearity of driving module in micro-gyroscope can be analyzed.

\section{Design of measuring system based on computer vision technology}

Computer micro vision system (CMVS) is a typical photoelectric measurement technology. It possesses many excellent characters such as non signal disturb, high distinguish ratio, accurate pitch position etc ${ }^{2}$, and is very suitable for measuring the vibration characteristic parameter for the driving module in micro-gyroscope. In most accessible literature, CMVS applied in vibration detection for micro-gyroscope is mostly focus on combining with stroboscopic imaging technology. ${ }^{3-5}$ Abiding by the principle that measuring method should matches and serves the test objective and their parameter peculiarity to measure, high-speed vidicon is used to obtain motion video of the driving module in testing process for study on dynamic characteristic of micro-gyroscope. After that, effectual image processing of computer vision technology is used to achieve the time-related motion curve, and herefrom, dynamic characteristic parameter can be analyzed and calculated.

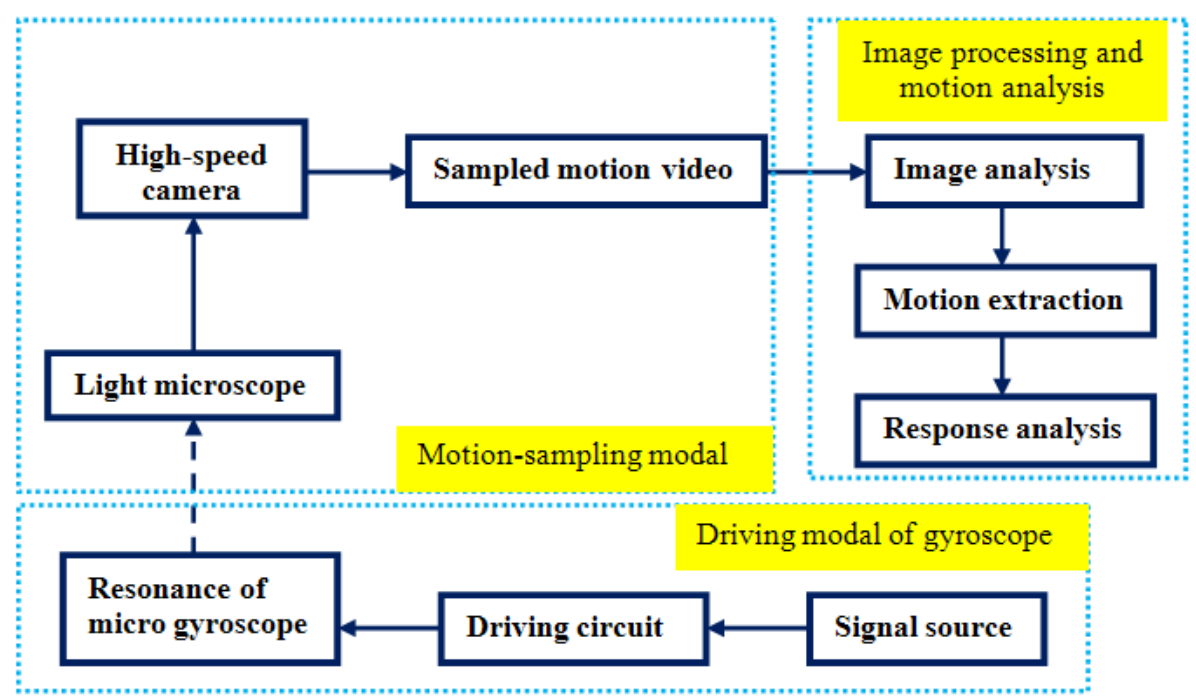

Fig.1 function block diagram of computer vision measuring system (CVMS)

\subsection{Combinations for computer vision measuring system}

The combined computer vision measuring system (CVMS) is shown as Fig.1. This system contains three function modules: driving module of micro-gyroscope, motion video obtaining module, image processing and motion parameter extracting module. Functions of driving module are to realize electrostatic driving in microstructure in micro-gyroscope, and furthermore to control the vibration to a balanced state. Motion video obtaining module consists of a light imager microscope 
containing a halogen lighting system, model of ZEISS Imager A2m, and a high-speed vidicon, model of Photron FastCAM SA4. With the incidence light through the packaging surface of micro-gyroscope, the vibration of driving comb can be observed clearly in a proper magnifying situation. With the seamless connect between microscope and high-speed vidicon by camera lens interface at the ocular end, images from the microscope can be wholly recorded by CCD sensor of the high-speed vidicon. In this way, the motion status of the

\subsection{Calibration of vision measuring system and related settings}

Calibrating for the vision measuring system is the basic preparation for CVMS. To calibrate the vision measuring system is to make certain the corresponding relationships between the position of imaging point in digital image and the actual location in microstructure for observed objects, and the relationships between the number of pixels occupied by observed objects and their actual size. The motion targets to measure in micro-gyroscope are focused on the driving detecting comb and their joint components such as bow-like driving beam which are vibrating in 2D plane. In this plane, the observed targets are vertical to the optical axis of imaging system, and the optical path of imaging is very short. So the imaging system used for vibration measuring for micro-gyroscope can be guaranteed as a pinhole camera (fitting in with the central projection relations). In this way, measuring precision error produced by imaging geometry distortion or imaging noise can be neglected. ${ }^{6}$

The observed motion targets is focused on driving detecting comb and their joint components such as bow-like driving beam, driving outer frame which their structure dimension has been surveyed accurately. So calibration of this vision measuring system can be carried out and the calibrating value can be defined by self-reference calibration approach ${ }^{7}$ in which the cor- microstructure in gyroscope can be recorded and saved as an AVI file under a suitable high sampling frequency by high-speed vidicon. Images stored in the AVI file can be processed, analyzed to identify and locate the motion objects by image processing and motion extracting module. By this effective image processing and motion analyzing, the time-related displacement curve of this recorded motion can be extracted, and the vibration characteristic parameter can be further analyzed for the driving module in micro-gyroscope.

responding relationship between actual dimensions and the sizes of pixels occupied by these structures in image is calculated from the known parameters of these micro-structures in micro-gyroscope.

Frame frequency of the high-speed vidicon set to shoot video of the vibration in micro-gyroscope should be considered synthetically, for the frequency of this vibration is very high (about $4000 \mathrm{~Hz}$ ). In evidence, higher the frame frequency of the vidicon set, more entire and more exact the video could mirror the actual motion of the high-speed vibration, and more correct is the obtained consults by means of image processing method. However, to increase the frame frequency will decrease obviously the exposure time and light intensity for the image, and it will reduce the image quality and might cause the analyzed results fulfilled by much noise. In order to obtain the optimal image quality, frame frequency of the vidicon should be decided through a great deal of experiments, adequately considering the inherent frequency of micro gyroscope and lighting conditions of the imaging system. Experimental results prove that the optimal frame frequency is about 30000 40000 fps while the inherent frequency of micro-gyroscope is about $4000 \mathrm{~Hz}$.

\section{Image processing and analysis of CVMS}

The AVI motion video file shot by high-speed vidicon is in fact a series of static images whose time interval is just the reciprocal of frame frequency of vidicon setting. 
Every frame of image expresses and corresponds to a certain instantaneous position of the vibration in micro-gyroscope at different time. The kernels of CVMS in motion measuring of micro-gyroscope is to obtain the related motion property from image sequences through proper image processing, right target identifying, locating accurately, and motion parameter extraction exactly.

Generally speaking, image processing called by a joint name in CVMS includes three aspects distinguished and related each another: image processing, image analysis and image comprehending ${ }^{8}$. Image processing emphasizes the transform between different images, through processing or treating beforehand at pixel level to meet the requirement of target identifying. Image analysis, being actually a process from image to data, emphasizes the target detection and measuring to construct a new image description according to the obtained image information in anterior image processing step. Image comprehending, based on the outcomes of image analysis, accentuates returning to the original physical meaning of targets in actual scene by further study on property of targets and their relationship in a same image or between different images.

Based on geometric and gray specialty analysis for the motion image of the vibration in micro-gyroscope, we take this technology line to process the obtained video: Firstly, the motion targets of the vibration are chose and limited in a suitable size windows in the datum image, and target identifying, locating, position parameter extracting are accomplished for one image in a certain area which is ascertained according to the vibration part in the micro-gyroscope. And then, the same algorithm operations are processed to all the frames of image contained in the motion video orderly. When this processing procedure for all the frames in the motion video has finished, the displacement curve with time-variation of the micro-gyroscope vibration comes out. The concrete process can be described as below:

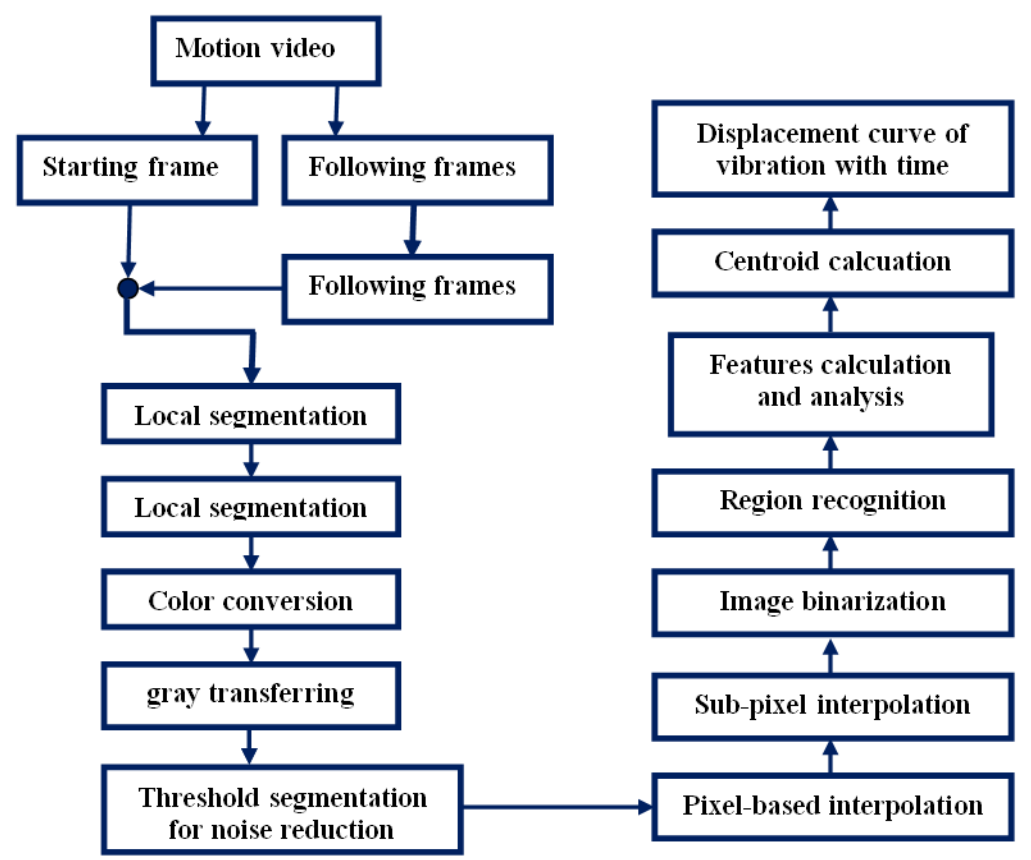

Fig.2 algorithm flow chart of CVMS 
(i) In procedure of image processing, it is indeed necessary to accomplish color-space transformation, noise-reduced threshold segmentation ${ }^{9}$, fundamental image interpolation, etc. Since the size range of the motion targets in micro-gyroscope is on micron level, their imaging in the obtained image occupies a few pixels, it is also necessary to refine the coarse pixels of primary images to sub-pixel representing according to the primary gray information. In this way, targets in image are located in sub-pixel precision. And then, binary image can be carried out on the base of anterior processing to provide for next step.

(ii) The procedure of image analysis aims at the binary image to take more processing. In this procedure, the Freeman method is applied to differentiate connected regions and non-connected regions in one image. And then, shape features of those connected regions are calculated and analyzed, and the centroid coordinates of the largest region can be fixed on by freeman formula.

(iii) In procedure of image comprehending, each frame of image in the video should be processed and analyzed by the same procedure of step1 and step 2. Every image corresponds to a certain time while the vibration under way, and one of the centroid coordinates can be acquired from the processing result for this image. With the value of frame time as abscissa $t$, and the value of centroid coordinate $y$ as ordinate, one point of the displacement of the motion target relative to time is confirmed, and all of the vibration displacements of micro-gyroscope can be obtained from all of the images in motion video. Further, the data points can be constituted by B-spline data fitting data to get a time-variation vibration curve for the vibration in micro-gyroscope.

The algorithm flow chart of CVMS is $\operatorname{shown}^{10}$ as Fig.2.

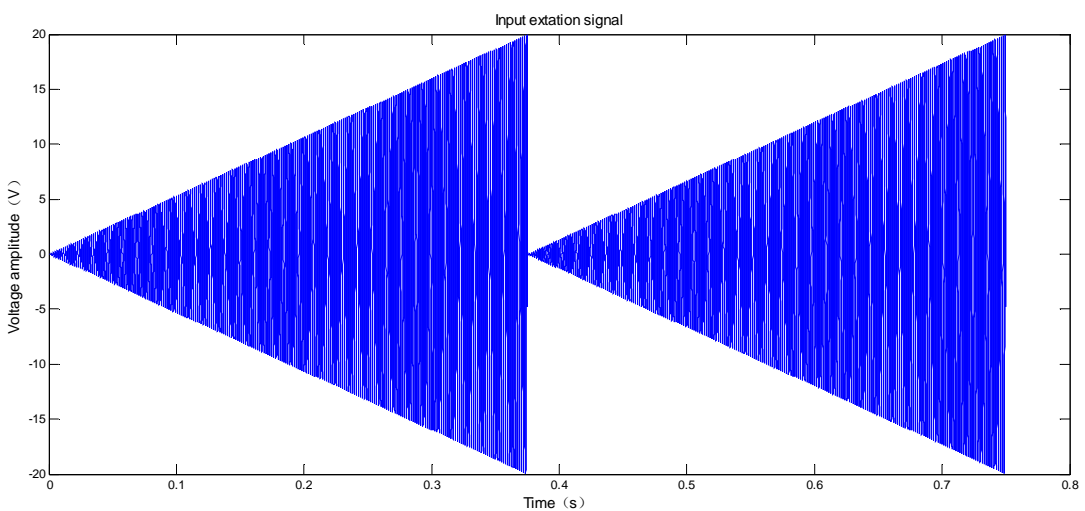

Fig.3 amplitude modulating excitation voltage signal ( $\mathrm{f}=3980 \mathrm{~Hz})$

\section{Linearity testing and analysis for micro-gyroscope driving module}

An essential feature of MEMS gyroscope is the distinct different between two stiffness bolstering vibration mass in $\mathrm{x}$ axis and in $\mathrm{y}$ axis directions, whether for driving module or for detecting modal. Stiffness in $\mathrm{x}$ axis direction for driving module, and stiffness in y axis direction for detecting module must be designed very smaller than stiffness in their perpendicular direction in which their bolstering are almost rigid. This special mechanical configuration can well eliminate mode coupling between the two modules. That is say the two vibration systems should possess good linearity in their 
libration directions. But in their performing situation, some facts will bring some un-neglectable nonlinear influence to the vibration system of gyroscope such as microscale effect, air damping, fluid-solid coupling effect, nonlinearity of electrostatic field, etc. In this

\subsection{Influence testing for driving signal to the amplitude of vibration}

Linearity of the driving module of gyroscope can be detected by parameter testing between displacement amplitude of vibration response and amplitude of the excitation signal based on the theory character relationship between response and its excitation signal for single-degree-of-freedom linear vibration system. Method of parameter testing is to exert the driving circuit of micro-gyroscope an excitation signal which frequency is constant and voltage amplitude varies from dissertation, we try to detect and evaluate the linearity of vibration system of driving module in micro-gyroscope, based on examining the relationship between the amplitude of vibration curve and the amplitude of driving voltage signal by CVMS method. ${ }^{10}$ $0 \mathrm{~V}$ to $20 \mathrm{~V}$ linearly, to shoot the vibration video, and get the time-related displacement curve by CVMS. And then, linearity of driving module can be studied based on this curve $\mathrm{e}^{11}$. The specific procedures are as follows:

(i) Construction of driving voltage signal: frequency of this driving signal is set to equal to the inherent frequency of driving module, here it is $3980 \mathrm{~Hz}$. The voltage amplitude varies linearly from $0 \mathrm{~V}$ to $20 \mathrm{~V}$ within $0.375 \mathrm{~s}$. This change period is set not be longer than half of the sampling time of vidicon. Fig.3 gives the oscillogram of this signal.

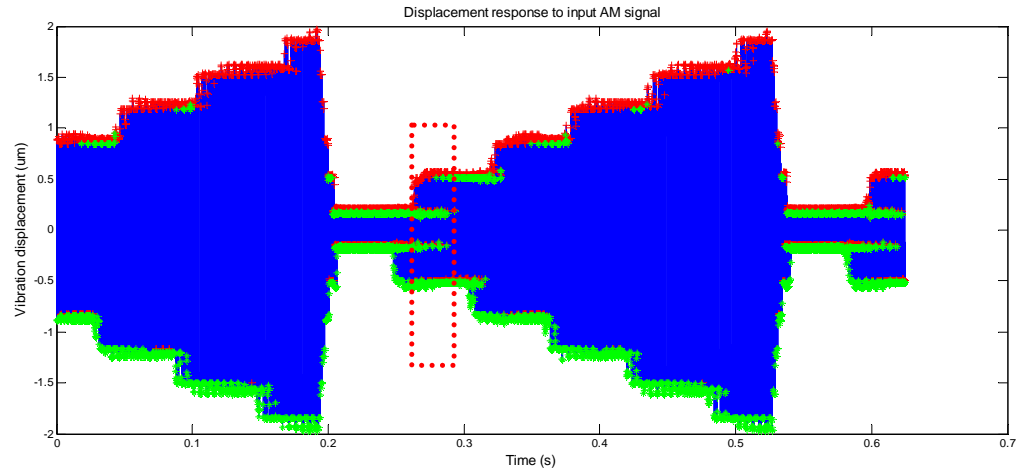

a) The development trend of gyroscope amplitude

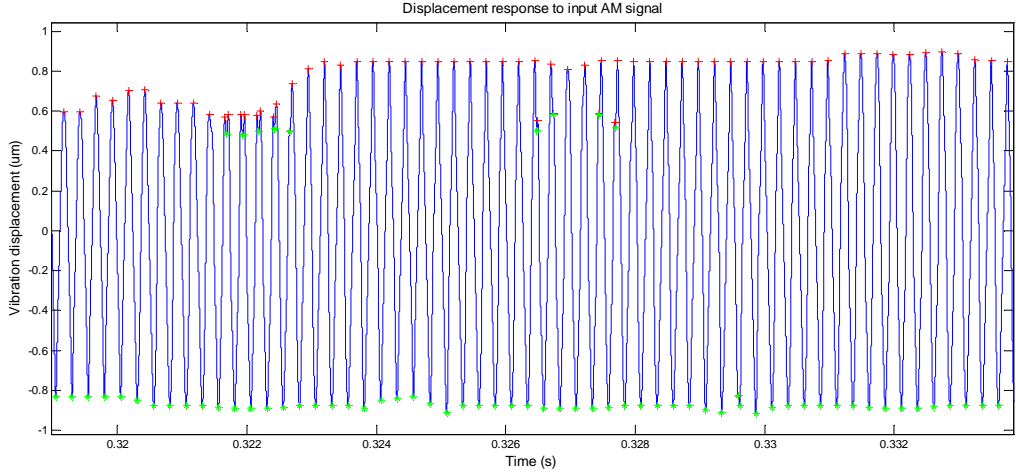

b) Part-enlarged picture of response amplitude curve

Fig.4 displacement response to input signal obtained by CMVS

(ii) Exert this driving voltage signal to the drive

circuit of micro-gyroscope, observe and shoot 
the motion video for vibration of the driving detecting comb in driving module. The video time should be longer than two times the change period of the driving voltage signal.

(iii) Process the vibration video by mean of CMVS, to obtain the vibration displacement time-variation curve for the driving module.

(iv) Contrast to analyze the displacement time-variation curve and the driving voltage signal, investigate and study relationship between amplitude of vibration displacement and amplitude of the driving voltage signal, to found data relationship between the two amplitudes.

(v) Linearity of driving module in micro-gyroscope can be researched based on this data relationship, and maximum variation of amplitude, linear least squares estimate, maximum deviation can be counted.

\subsection{Vibration response to the driving voltage sig-}

nal

Fig.4 gives the vibration response of the driving module vibration system to the driving voltage signal ${ }^{10}$. Fig.4a shows the vibration displacement time-variation curve processed by and gotten as a result of CVMS. In this figure, two intact varying periods of the displacement curve shows accurately the corresponding relation to the scan period of driving voltage signal. For the high-frequency vibration, a great deal of data points is generated and heap up in this figure. Fig. $4 \mathrm{~b}$ gives a part-enlarged picture of the curve in Fig.4a to observe the waveform details in an observing window.

Compare the amplitudes of vibration displacement in Fig.4a and the amplitudes of driving voltage signal in Fig.3 on a same starting time point for their synchronous variation character to time. In this way, corresponding data relation between the amplitude of vibration displacement and the amplitudes of driving voltage signal can be established as shown in Fig.5.

\subsection{Linearity analysis for vibration system of mi- cro-gyroscope}

Least square fitting method is most commonly used in datum line fitting for linearity assessing. Fit the data of corresponding amplitudes shown in Fig.5 by least square fitting method, a datum line to assess the linear relationship between the vibration amplitude response and its driving voltage excitation can be expressed as:

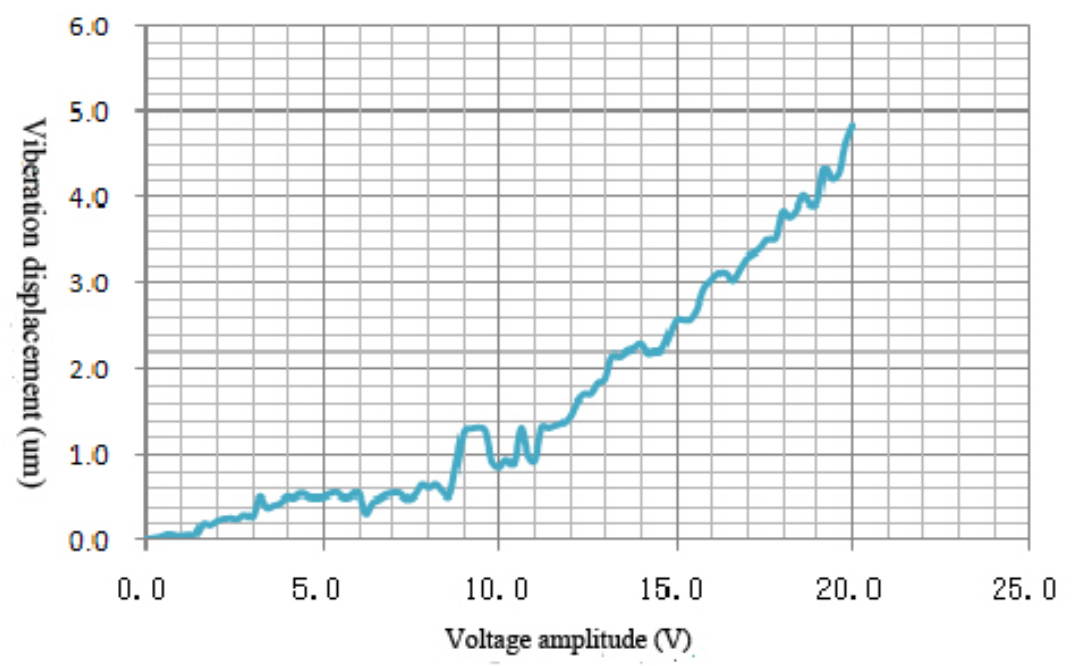

Fig.5 corresponding relationship between response amplitude and excitation voltage 
$\mathrm{F}=0.2178 \mathrm{~V}-0.6184$, it is shown in Fig.6.

Based on this assessing datum line, the maximum deviation of sampling data can be calculated as +1.1174 um, while the amplitude of driving voltage signal is $20 \mathrm{~V}$. With the output scale range of vibration amplitude is 6um, the least squares linearity (L) of the driving module can be calculated as: $\mathrm{L}=1.1174 / 6.0=18.62 \%$.

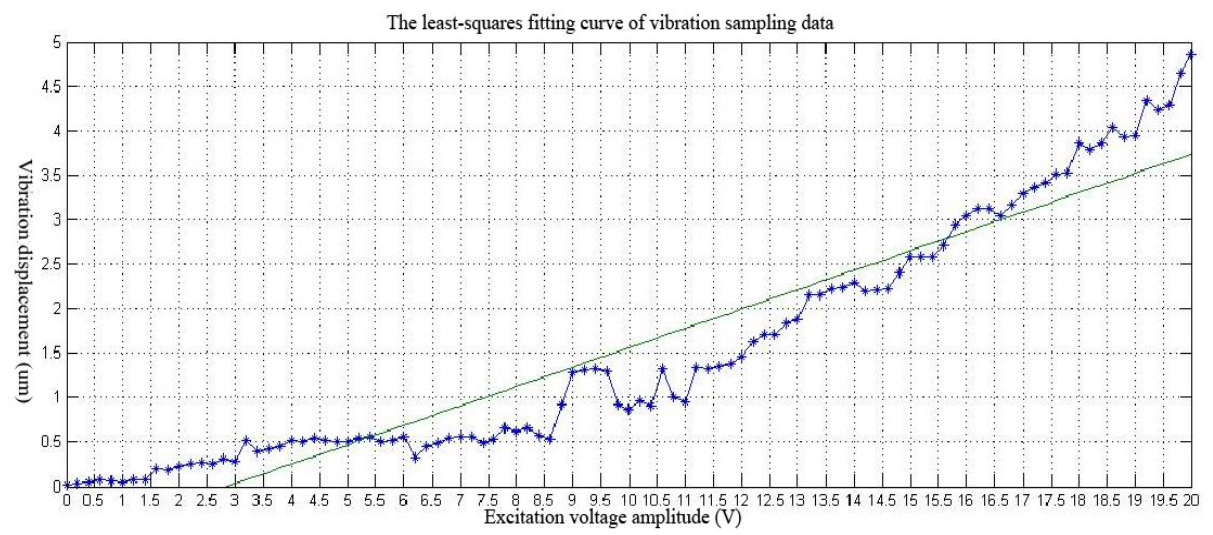

Fig.6 linear least squares estimate between vibration amplitude and input voltage

\section{Conclusions}

Computer vision measuring system based on high-speed vidicon is an effective solution for motion detecting and dynamic characteristic analyzing for micro-gyroscope in working state. Vibration displacement time-related curve can be obtained directly by CVMS from the actual behaving video of the vibration targets. In this way, more dynamic characteristic parameter for the vibration system of driving module in micro-gyroscope can be analyzed and calculated. CVMS method is used to assess the linearity of response and its excitation for driving module in micro-gyroscope at working condition. Conclusions by this way could be proved efficient and accurate.

\section{References:}

1. Ke-jun XU, Yong-bao CHEN, Chong-wei ZHANG, The common technology of automatic detection and instruments (Tsinghua University Press, 2000),pp.171-175.

2. Bo-xiong Wang, Fei-fan Chen, Ying Dong, Micro-nano detection technology (Tsinghua University Press, 2006).
3. S. Park, Adaptive control strategies for MEMS gyroscopes, Doctoral dissertation (Berkeley, CA, 2000).

4. Shu-Ang Zhou, On forces in micro-electromechanical systems, Int. J. of Engineering Science, 41(2003) pp.313-335.

5. Da-chao Jia, Ya-lin Feng, Xing Fu, MEMS dynamic testing technique, MEMS Device \&Technology, 4(2005) pp.188-192.

6. Qi-feng Yu, Yang Shang, Videometrics:Principles and Researches, Science Press (2009) pp.16-19.

7. Chang-chun Li, Study and realization of camera calibration technology in machine vision, Nanjing University of Aeronautics and Astronautics, (2009).

8. Rang-pei Qin, Image processing and graphical interface programming by MATLAB, Publishing House of Electronics Industry, (2009).

9. Tao Wu, Kun Qin. Comparative Study of Image Thresholding Using Type-2 Fuzzy Sets and Cloud Model, International Journal of Computational Intelligence Systems, Suppl.1 (2010) pp.61-73.

10. Yong Zhou, Study on dynamic characteristic and its detection technology for MEMS gyroscope, Beijing Institute of Technology, (2011).

11. J. Wesley, HINES, Alexander USYNIN. Current Computational Trends in Equipment Prognostics, International Journal of Computational Intelligence Systems, 1(1)(2008) pp.94-102. 